\title{
Analytical Modeling and Control of Rectilinear Snake Robots
}

\author{
Dr. S.V. Saravanan and S. Sindhuja \\ Assistant Professor, Department of EEE-M, Amet University, Chennai, \\ Research scholar, Department of EEE-M, Amet University, Chennai, \\ svsaravanan@gmail.com, sindhujatce@gmail.com
}

\begin{abstract}
This paper deals with the analytical modeling and control of rectilinear snake robots. During recent times snake robots have created much interest among researchers. The rectilinear pattern gait is one of the four biological snake locomotion modes. Rectilinear snakes have been widely used in rescue operations especially in rough terrains especially in narrow spaces where human intervention is not easy. Computational analysis of rectilinear motion is done using MATLAB.

Keywords - rectilinear; PID; friction; spring-mass system; dynamics
\end{abstract}

\section{INTRODUCTION}

Bio-inspired robots have been used in many practical applications There are many recent successful attempts to make crawling robots. The snake robots are such robots which provide advantageous properties in hard to reach areas because of its good skeletal structure. Research on snake robots is inspired by the robust motion capabilities of biological snakes. The snake motion is very stable because during its motion it has body parts in the contact with the surface. This area of research is in most cases only in theoretical level since the snake-like robots are very difficult to design and control.

. The biological snake locomotion are:
a. Lateral undulation
b. Concertina locomotion
c. Rectilinear crawling
d. Sidewinding

The rectilinear locomotion mode is usually the pattern gait by heavy snakes which are not possible to move by undulation. This rectilinear locomotion mode is used to achieve desired model of snake robots. The mathematical model is developed using MATLAB. Theoretical level analysis has been done where the model consists of identical masses and passive bonds.

\section{SPRING-MASS MODEL}

The rectilinear locomotion can be realized using the spring-mass model consisting of massless spring and dampers between segments of snake robots. Figure 1 shows the simplified model of the snake consisting of $\mathrm{N}$ consecutive masses of the weight $\mathrm{m}$.

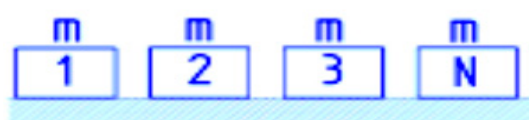

Figure 1. Simplified model of the snake
For having a simplified analysis we simplify the above $\mathrm{N}$ mass model as a two mass model as shown in Figure 2 and spring damper $\mathrm{N}$ mass model is shown in Figure 3.

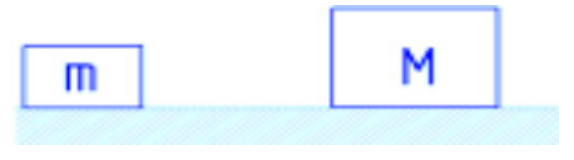

Figure 2. Two mass model of the snake

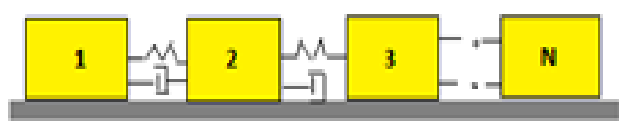

Figure 3. Spring- damper $\mathrm{N}$ masses mechanical system

This Spring-damper model depicts the snake rectilinear motion. Next, the same propulsive force affects $n$-th moving mass; therefore each mass moves the same time t. The interaction between snake robot and dry surface on which it moves is modeled as Coulomb friction. The example of motion sequence of 5-mass mechanical system is shown on the Figure 4.

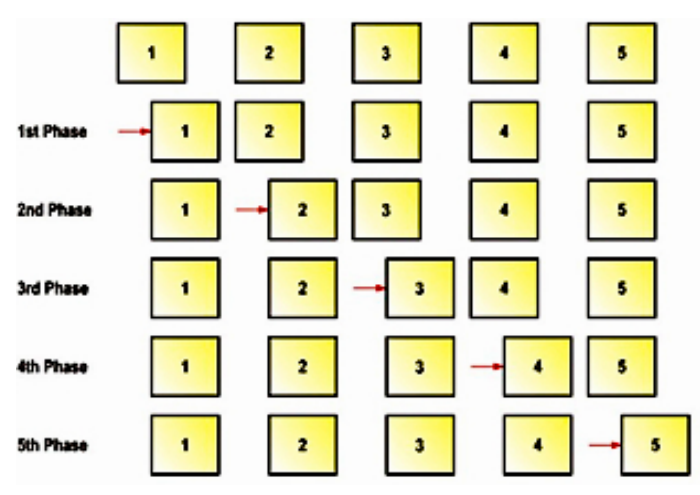

Figure 4. Sequence of snake robot rectilinear motion

As can be seen on the Figure 4, during one motion cycle still only one mass moves while other are at rest. The actuators between static masses operate so, that static masses behave as one mass according to Figure 6 . 


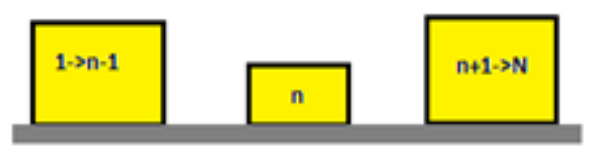

Figure 5. Simplified model of N-mass mechanical system during moving of $\mathrm{n}$-th mass

It is obviously that $\mathrm{n}=2,3 \ldots \mathrm{N}-1$. On the outer masses (first and last mass) acts the same propulsive force as on the inner masses. Before further analysis will be determine whether occurs movement of adjacent masses of $n$-th mass because of spring and damper forces.

\section{A. Computational Analysis}

For computational analysis purpose we further simplify as Spring-damper two masses system.

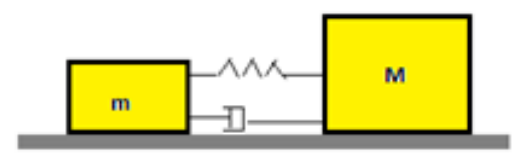

Figure 6. Simplified 2-mass mechanical system

The advantage of this model in comparison with model without passive bonds (springs and dampers) is that by suitable spring and damper coefficients can be motion kinematic parameters affected. The conditions of rectilinear motion of our model are that the mechanical system consists of $\mathrm{N}$ masses with the same weight $\mathrm{m}$.

The equations of motion can be written as follows:

$$
\begin{array}{r}
m \frac{d^{2} x_{m}(t)}{d t^{2}}+b \frac{d\left(x_{m}-x_{M}\right)}{d t}+k\left(x_{m}-x_{M}\right)=F_{p}-F^{1}{ }_{f c} \\
\ldots .(1) \\
M \frac{d^{2} x_{M}(t)}{d t^{2}}+b \frac{d\left(x_{M}-X_{m}\right)}{d t}+k\left(x_{M}-x_{m}\right)=-F_{p}+F^{2}{ }_{f c}
\end{array}
$$

Where $\mathrm{x}_{\mathrm{m}}, \mathrm{x}_{\mathrm{M}}, \mathrm{m}, \mathrm{b}, \mathrm{k}, \mathrm{F}_{\mathrm{p}}$ and $\mathrm{F}_{\mathrm{fc}}$ are displacement of moving mass, displacement of static mass, weight of moving mass, coefficient of damper, stiffness of spring, propulsive force and Coulomb friction force, respectively.

Taking Laplace transform of both equations,

$$
\begin{gathered}
m s^{2} x_{m}(s)+b s x_{m}(s)-b s x_{M}(s)+k x_{m}(s)-k x_{M}(s) \\
=F_{p}(s)-F^{1}{ }_{f c}(s)
\end{gathered}
$$

$$
\begin{gathered}
M s^{2} x_{M}(s)+b s x_{M}(s)-b s x_{m}(s)+k x_{M}(s)-k x_{m}(s) \\
=-F_{p}(s)+F_{f c}^{2}(s)
\end{gathered}
$$

Grouping terms $\mathrm{x}_{\mathrm{m}}$ and $\mathrm{x}_{\mathrm{M}}$ separately,

$$
\begin{aligned}
\left(m s^{2}+b s+k\right) x_{m}(s)- & (b s+k) x_{M}(\mathrm{~s}) \\
= & F_{p}(s)-F^{1}{ }_{f c}(s) \\
& \\
\left(M s^{2}+b s+k\right) x_{M} & -(b s+k) x_{m} \\
& =-F_{p}(s)+F_{f c}^{2}(s)
\end{aligned}
$$

Writing equation in matrix form,

$$
\begin{gathered}
{\left[\begin{array}{cc}
m s^{2}+b s+k & -(b s+k) \\
-(b s+k) & M s^{2}+b s+k
\end{array}\right]\left[\begin{array}{l}
x_{m} \\
x_{M}
\end{array}\right]=} \\
{\left[\begin{array}{c}
F_{p}(s)-F_{f c}^{1}(s) \\
-F_{p}(s)+F_{f c}^{2}(s)
\end{array}\right]}
\end{gathered}
$$

Solving for $\mathrm{x}_{\mathrm{m}}$ and $\mathrm{x}_{\mathrm{M}}$ by using Cramer's rule we get,

$$
\begin{aligned}
& x_{m}=\frac{F_{p}(s)-F_{f c}^{1}(s)}{m s^{2}+\frac{b s}{M}\left(\frac{m}{M}+1\right)+\frac{k}{M}\left(\frac{m}{M}+1\right)} \\
& x_{M}=\frac{-F_{p}(s)+F_{f c}^{2}(s)}{M s^{2}+\frac{b s}{m}\left(\frac{M}{m}+1\right)+\frac{k}{m}\left(\frac{M}{m}+1\right)}
\end{aligned}
$$

\section{B. $\quad$ Analysis of nth moving mass}

For inner $n$-th moving mass $(n=2,3 \ldots N-1)$ can be used simplified model according to Figure 7.

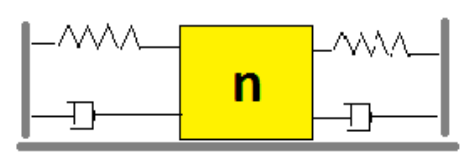

Figure 7. Simplified model of nth moving mass

For n-th moving mass equations of motion can be written follows

$$
\begin{aligned}
\frac{d x_{n}(t)}{d t^{2}}+\frac{b_{n-1}+b_{n}}{m} & \frac{d x_{n}}{d t}+\frac{k_{n-1}+k_{n}}{m} x_{n}(t) \\
& =\frac{F_{P 1}+F_{P 2}-F_{f c}^{n}}{m}
\end{aligned}
$$

Taking Laplace transform,

$$
X(s)=\frac{F_{p 1}+F_{P 2}+F_{f c}^{1}}{m} \frac{1}{s\left(s^{2}+\frac{2 b}{m} s+\frac{2 k}{m}\right)}
$$


The term $\left(s^{2}+\frac{2 b}{m} s+\frac{2 k}{m}\right)$ represents second order dynamic system. The objective is that the system should not have oscillating character. In order to achieve no oscillating character of variable $x(t)$, from the dynamic system theory it is known that mentioned term has to have one double root $s k$. The root is

$$
s_{k}=-\frac{\left(b \pm \sqrt[2]{b^{2}-2 k m}\right)}{m}
$$

The equation (11) can be simplified as,

$$
X(s)=\frac{F_{P 1}+F_{P 2}-F_{f c}^{n}}{m} \frac{1}{s\left(s+s_{k}\right)^{2}}
$$

By distribution of term $\frac{1}{s\left(s+s_{k}\right)^{2}}$ into partial fractions we obtain

$$
\frac{1}{s\left(s+s_{k}\right)^{2}}=\frac{A}{s}+\frac{B}{\left(s-s_{k}\right)^{2}}+\frac{C}{\left(s-s_{k}\right)}
$$

By further solving we obtain coefficients $A, B, C$.

$$
\begin{gathered}
A=\frac{1}{s^{2}{ }_{k}} \\
B=\frac{1}{s_{k}} \\
C=-\frac{1}{s^{2}}{ }_{k}
\end{gathered}
$$

\section{PID Controller}

A proportional-integral-derivative controller (PID controller or three term controller) is a control loop feedback mechanism widely used in industrial control systems and a variety of other applications requiring continuously modulated control. A PID controller can be used for regulation of speed, temperature, flow, pressure and other process variables.

Here a PID controller is used for controlling displacement and speed of rectilinear snake robots. First step input is applied to the transfer function to study the characteristics of the system. Then by manual tuning the closed loop response of the system is completed.

\section{Simulation results}

Simulation results using MATLAB are shown from Figures 8-13

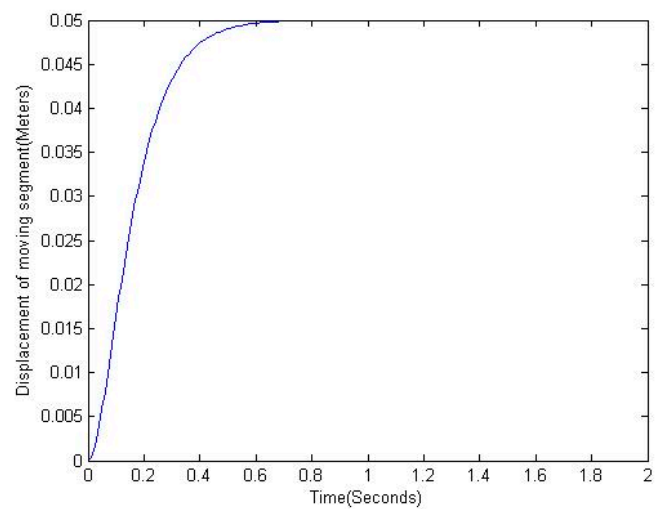

Figure 8. Displacement of the moving mass

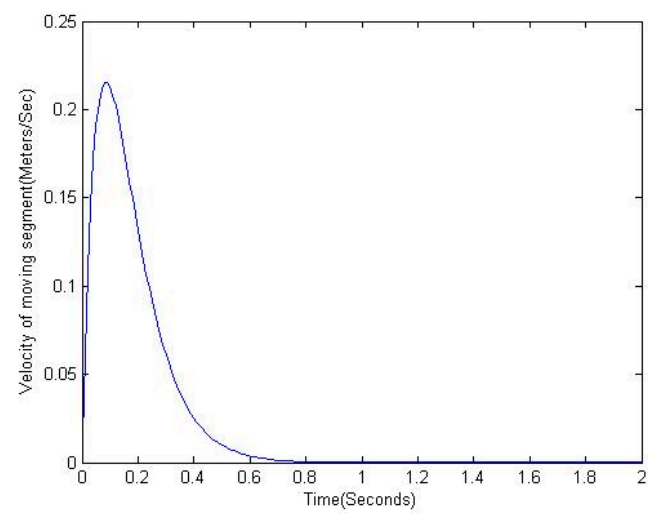

Figure 9. Velocity of the moving mass

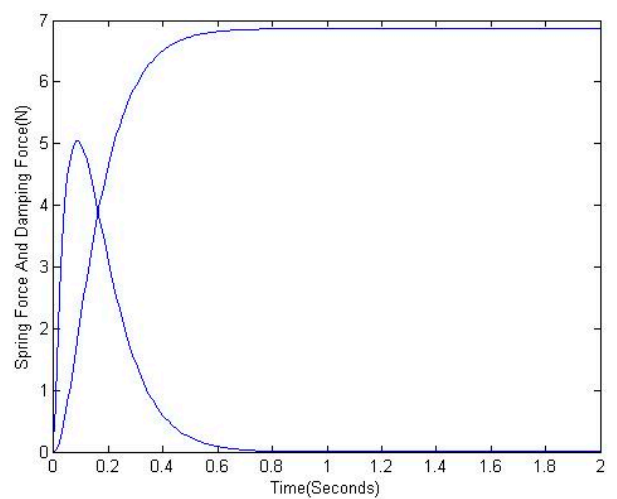

Figure 10. Spring and damper forces

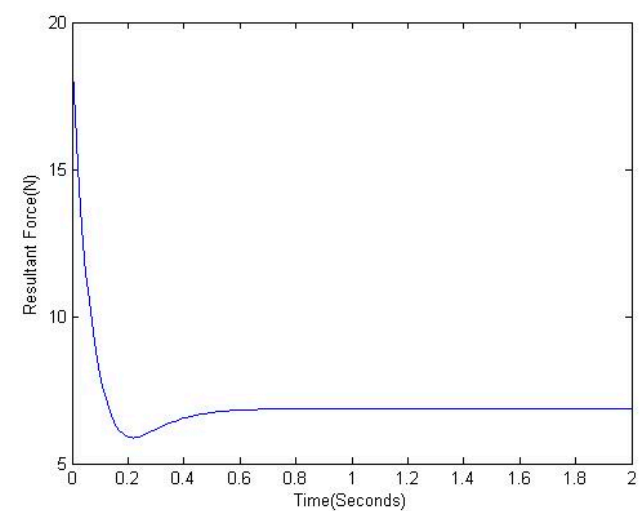

Figure 11. Resultant force affecting the mass 


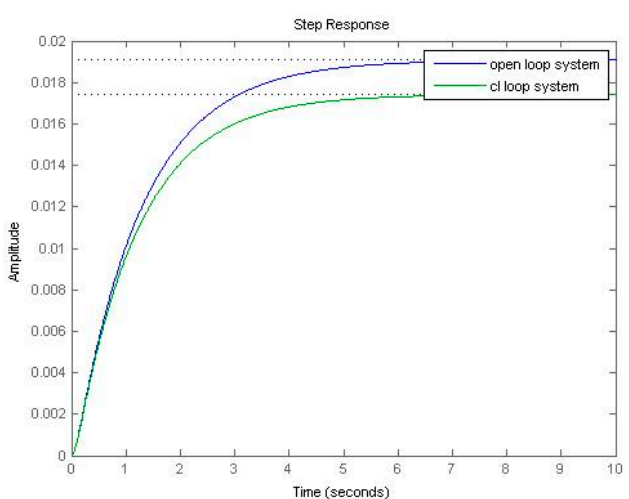

Figure 12. PID Control of Spring - Mass system

\section{CONCLUSION}

In this paper the snake rectilinear motion was investigated. Snake body was replaced by identical masses which represent the segments of snake robot. In this paper the snake rectilinear motion mathematical model is established and the displacement and velocity of $\mathrm{n}$-th moving mass is derived. Subsequently, the forces affecting the $n$-th moving mass are shown in the graphs. The control of the moving segment is being studied and the results infer that there is $5.5 \%$ reduction of settling time, which results in effective control. We would extend this work in future for motion analysis of rectilinear snakes in random surface.

\section{REFERENCES}

[1] T. Dowling, K. J. (1997), Limbless locomotion: Learning to crawl with a snake robot, Doctoral Thesis, Carnegie Mellon University, pp. 3-7.

[2] Transeth, A. A., Pettersen K. Y., Liljebäck, P. (2009), A survey on snake like robot modeling and locomotion, Robotica, Vol. 27, pp. 999 - 1015.

[3] R. Grepl, "Mechatronic system modeling in Matlab / SimMechanics", ISBN 978-80-7300-226-8, Prague 2007.

[4] Ghanbari, A., Fakhrabadi, M. S., Rostami, A. (2009), Dynamics and GA-Based Optimization of rectilinear Snake Robot, Intelligent Robotics and Applications, pp. $613-622$.

[5] Lissmann, H. W. (1949), "Rectilinear locomotion ion in a snake", Th e Journal of Experimental Biology, pp. 368 379.

[6] Virgala, I. (2012), Mechatronic approach to design of system imitating locomotion of snake in limited spaces, Doctoral Thesis, Technical University of Košice.

[7] A. Gmiterko, P. Šarga, D. Hroncová, "Dynamic systems theory", ISBN 978-80-553-0603-2, Košice, Slovakia, 2010.

[8] A. Gmiterko, I. Virgala "Simplified model of a snake rectilinear motion", 9th IEEE International Symposium on Applied Machine Intelligence and Informatics, pp. 307310, January 2011.

[9] I. Virgala, M. Kelemen, E. Prada and R. Surovec "Motion analysis of snake robot segment", 11th IEEE International Symposium on Applied Machine Intelligence and Informatics, January 31 - February 2, 2013.

[10] Ivan Virgala, Alexander Gmiterko, Peter Frankovský "Experimental Analysis of Friction Effects Affecting the Snake Robot Segment during Rectilinear Locomotion"
American Journal of Mechanical Engineering, 2013, Vol. 1, No. 7, 412-416

[11] F.L.Chernous'ko "The optimum rectilinear motion of a two-mass system" Journal of Applied Mathematics and Mechanics. Volume 66, Issue 1, 2002, Pages 1-7

[12] N.N.BolotnikT, Yu.FigurinaF.L, Chernous'ko "Optimal control of the rectilinear motion of a two-body system in a resistive medium" Volume 76, Issue 1, 2012, Pages 1-14

[13] N.N.BolotnikT, Yu.Figurina" Optimal control of the rectilinear motion of a rigid body on a rough plane by means of the motion of two internal masses" Volume 72, Issue 2, August 2008, Pages 126-135

[14] Nikolay Bolotnik, Felix Chernousko, Tatiana Figurina "Optimal Control of a Two-body: Vibration-driven Locomotion System in a Resistive Environment" IFACPapersOnLine, Volume 48, Issue 25, 2015, Pages 91-96

[15] Y. Peng, D. Vrancic, and R. Hanus. Anti-Windup, Bumpless, and Conditioned Transfer Techniques for PID Controllers. IEEE Control Systems, pages 48-57, 1996. B.3.3

[16] E.Kurtulmuş Locomotion and Control of a modular snake like robot 2010.

[17] "Modular snake robots: gaits," Biorobotics Laboratory: Carnegie Melon University, 2008. [Online]. Available: http://biorobotics.ri.cmu.edu/projects/modsnake/newwebsi te/gaits/. [Accessed 15 July 2016].

[18] A. A. Transeth, Modeling and control of snake robots, Norwegian University of Science and Technology, 2008.

[19] A. A. Transeth and K. Y. Pettersen, "Developments in snake robot modeling and locomotion," in 9th International Conference on Control, Automation, Robotics and Vision. IEEE, 2006.

[20] P. Liljebäck and e. al., Snake robots: modelling, mechatronics, and control, London: Springer-Verlag, 2012. 